\title{
Evaluation of the Internal Nasal Valve using Computed Tomography Pre and Post Rhinoplasty and Its Correlation to Symptomatic Improvement

\author{
${ }^{1}$ Amr Gouda Shafik, ${ }^{2}$ Ahmed Mostafa Mohamed, ${ }^{1}$ Tahany Mohamed Rabie, ${ }^{1}$ Hussam Adel \\ Alkady
}

${ }^{1}$ Department of Otorhinolaryngology, ${ }^{2}$ Department of Diagnostic Radiology ,Faculty of medicine, AinShams University

Corresponding author: Hussam A. Alkady, Mobile: 01111390530; Email: hussamadel@ hotmail.com

\begin{abstract}
Background: The INV area is the narrowest area of a nasal cavity, which is formed by the nasal septum, the caudal border of the upper lateral cartilage ULC, the head of the inferior turbinate, and the pyriform aperture and the tissues that surround it. Accurate evaluation of the INV is important to the workup for repair of problems that involve this area. The initial evaluation of the nasal valve occurs with the clinical examination, anterior rhinoscopy, the Cottle maneuver, and endoscopy, which all are used clinically to assess nasal airflow.

Objective: This study aims to evaluate the internal nasal valve by using reformatted (CT) scans of the nasal airway pre and post rhinoplasty and its correlation to symptomatic improvement according to (NOSE) scale.

Patients and Methods: This prospective study of twenty patients who were subjected to rhinoplasty at ENT departments of Ain-shams university hospitals.

Results: This study included 20 cases of age's ranges from 20 to 30 years. The mean age among study cases was $23.8 \pm 3.22$. There were 14 males and 6 females. So, the majority of cases (70\%) were males. In our study, we detected that there is no significant correlation between CT scans analysis of the INV pre and postrhinoplasty and there is no significant correlation between CT scans analysis of the INV and the NOSE score pre and post-rhinoplasty. On the other hand, there is high significant correlation between NOSE score pre and post-rhinoplasty.

Conclusion: In our study, we detected that there is no significant correlation between CT scans analysis of the INV pre and post-rhinoplasty and there is no significant correlation between CT scans analysis of the INV and the NOSE score pre and post-rhinoplasty.

Keywords: External nasal valve - internal nasal valve - nose obstruction symptomatic evaluation
\end{abstract}

\section{INTRODUCTION}

The airflow resistance provided by the airways during breathing is essential for good pulmonary function. Nasal airway resistance accounts for more than $50 \%$ of total airway resistance, most of this resistance occurs in the anterior part of the nose which called the nasal valve ${ }^{(\mathbf{1})}$.

In 1903, the nasal valve was originally described by Mink. It is divided into external and internal portions. The external nasal valve is formed by the columella, the nasal floor, and the nasal rim (or caudal border of the lower lateral cartilage LLC). The internal nasal valve INV area is the narrowest area of a nasal cavity, which is formed by the nasal septum, the caudal border of the upper lateral cartilage ULC, the head of the inferior turbinate, and the pyriform aperture and the tissues that surround it ${ }^{(2)}$.

Collapse of the internal nasal valve is a common finding in patients who present with nasal airway obstruction. Although this can be a congenital condition, weakness of the internal nasal valve can also occur with nasal aging, following traumatic injury, and even more commonly because of prior nasal surgery secondary to rhinologic surgery ${ }^{(3)}$.
Correction of internal valve collapse must be done during both functional rhinoplasty, which may include inferior turbinectomy, septoplasty, and placement of spreader grafts which is one of the most effective means of correcting internal valve collapse ${ }^{(4)}$.

Accurate preoperative evaluation of the internal nasal valve is critical to the workup for reconstruction or repair of problems that involve this area. A CT scan of the internal nasal valve can provide the surgeon with improved anatomical information to assess that region ${ }^{(5)}$.

Computed Tomography (CT) has been proposed as an objective tool to measure internal nasal valve anatomy preoperatively and postoperatively. The acquired CT scans must be reformatted to obtain sections through the internal nasal valve at a more appropriate orientation by using reformatted coronal and sagittal CT scans, so the valve cross-sectional area and valve angle can be measured ${ }^{(6)}$.

\section{AIM OF THE WORK}

This study aims to evaluate the internal nasal valve by using reformatted (CT) scans of the nasal airway pre and post rhinoplasty and its correlation to symptomatic improvement according to (NOSE) scale. 


\section{PATIENTS AND METHODS}

This prospective study of twenty patients who were subjected to rhinoplasty at ENT departments of Ain-shams university hospitals. The study was approved by the Ethics Board of Ain Shams University.

Inclusion patient's criteria: Male and female patients age ranging from 20-50 years old. Nasal obstruction secondary to internal nasal valve collapse \pm inf. turbinate hypertrophy \pm deviated nasal septum. Exclusion patient's criteria: Sinusitis. Nasal polyposis. Nasal masses.

All patients were asked for permission to participate in the study to be assessed by CT scan of the nose pre-rhinoplasty and 3 months post-rhinoplasty by reformatted coronal CT scans of internal nasal valve area. It will be used at plane perpendicular to the anterior aspect of the acoustic axis. The acoustic axis is estimated on a sagittal reformatted image based on the results of Cakmak et al. ${ }^{(7)}$ who showed that the axis passes through the center of the nasal passage in an arc (fig 1). The internal nasal valve cross-sectional area and valve angle will be measured through a standardized section (1 cut immediately anterior to the head of the inferior turbinate) from the reformatted scans (fig. 2- fig. 5).

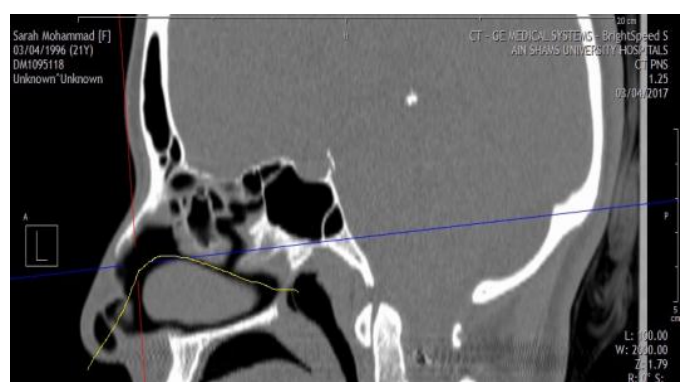

Figure (1): Sagittal reformatted computed tomographic view of the nasal cavity. The yellow line indicates the estimated acoustic axis and physiologic nasal airflow.

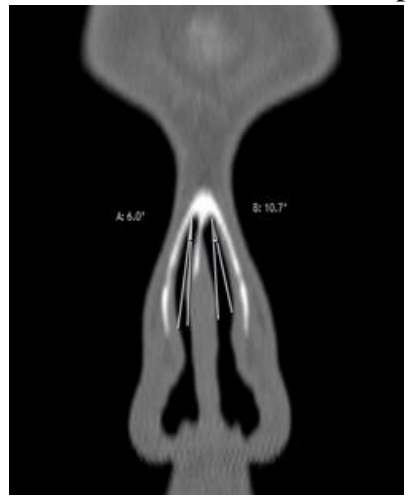

Figure (2): INV angle preoperative

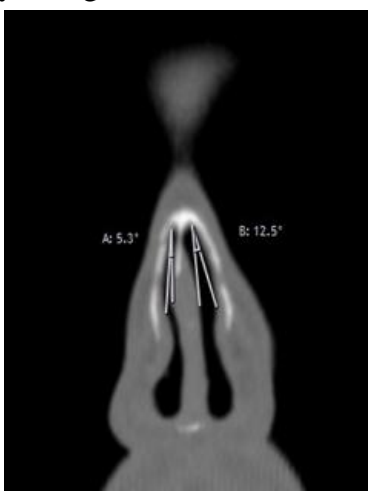

Figure (3): INV angle post-operative

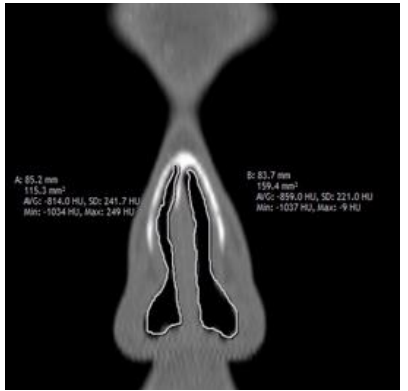

Figure (4): INV area preoperative

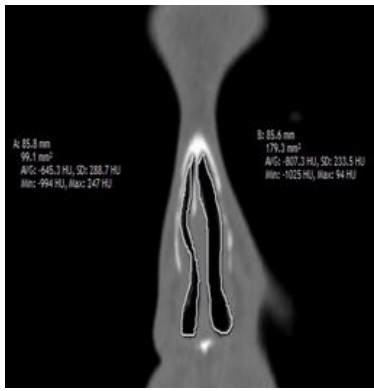

Figure (5): INV area post-operative

\section{RESULTS}

Table (1): Comparison between males and females as regard post-operative nasal score.

\begin{tabular}{|l|cc|cc|c|c|}
\hline \multirow{2}{*}{} & \multicolumn{4}{|c|}{ Sex } & \multirow{2}{*}{ P } & \multirow{2}{*}{ Sig } \\
\cline { 2 - 5 } & \multicolumn{2}{|c|}{ Male } & \multicolumn{2}{c|}{ Female } & \\
\cline { 2 - 4 } & Mean & $\mathbf{\pm S D}$ & Mean & $\mathbf{\pm S D}$ & & \\
\hline $\begin{array}{l}\text { Postoperative } \\
\text { nose score }\end{array}$ & 39.29 & 7.56 & 38.33 & 4.08 & .777 & NS \\
\hline
\end{tabular}

Table (2): Multiple regression to study independent factors affecting post-operative nose score.

\begin{tabular}{|l|c|c|c|c|c|}
\hline & $\begin{array}{c}\text { Regression } \\
\text { coefficient }\end{array}$ & P & Sig. & \multicolumn{2}{|c|}{$\begin{array}{c}\mathbf{9 5 . 0 \%} \text { CI for } \\
\text { regression coefficient }\end{array}$} \\
\hline Age & -1.05 & $\mathbf{. 0 3 3}$ & $\mathbf{S}$ & -2.003 & -.097 \\
\hline Sex & 1.239 & .678 & $\mathrm{NS}$ & -4.99 & 7.473 \\
\hline post INV area & -.0112 & .076 & $\mathrm{NS}$ & -.237 & .013 \\
\hline post INV angle & -.684 & .203 & $\mathrm{NS}$ & -1.77 & .410 \\
\hline
\end{tabular}

Table (3): Comparison between pre-operative and post-operative INV area.

\begin{tabular}{|l|c|c|c|c|}
\hline & Mean & Std. Deviation & P & Sig \\
\hline Pre INV area Lt. Side & 130.3 & 44.99 & \multirow{2}{*}{642} & \multirow{2}{*}{ NS } \\
\cline { 1 - 3 } Post INV area Lt. Side & 125.2 & 30.40 & & \\
\cline { 1 - 3 } Pre INV area Rt. Side & 138.66 & 60.67 & \multirow{2}{*}{613} & \multirow{2}{*}{ NS } \\
\hline Post INV area Rt. Side & 130.98 & 30.65 & & \\
\cline { 1 - 3 } Average pre INV area & 134.48 & 34.57 & \multirow{2}{*}{.431} & \multirow{2}{*}{ NS } \\
\hline Average post INV area & 128.09 & 24.79 & & \\
\hline
\end{tabular}

Table (4): Comparison between pre-operative and post-operative INV angles.

\begin{tabular}{|c|c|c|c|c|}
\hline & Mean & $\begin{array}{c}\text { Std. } \\
\text { Deviation }\end{array}$ & $\mathbf{P}$ & Sig \\
\hline Pre INV angel Lt. Side & 9.35 & 4.56 & \multirow{2}{*}{.683} & \multirow{2}{*}{ NS } \\
\hline Post INV angle Lt. Side & 9.85 & 3.22 & & \\
\hline Pre INV angel Rt. Side & 9.15 & 5.19 & \multirow{2}{*}{.170} & \multirow{2}{*}{ NS } \\
\hline Post INV angle Rt. Side & 10.75 & 3.73 & & \\
\hline Average pre INV angle & 9.25 & 2.25 & \multirow{2}{*}{.121} & \multirow{2}{*}{ NS } \\
\hline Average post INV angle & 10.30 & 2.93 & & \\
\hline
\end{tabular}


Table (5): Comparison between pre-operative and post-operative nose score.

\begin{tabular}{|l|c|c|c|c|}
\hline & Mean & $\begin{array}{c}\text { Std. } \\
\text { Deviation }\end{array}$ & P & Sig \\
\hline Pre-operative nose score & 67.50 & 7.864 & \multirow{2}{*}{.0001} & HS \\
\hline Post-operative nose score & 39.00 & 6.609 & & \\
\hline
\end{tabular}

\section{DISCUSSION}

The INV area is the narrowest area of a nasal cavity, which is formed by the nasal septum, the caudal border of the upper lateral cartilage ULC, the head of the inferior turbinate, and the pyriform aperture and the tissues that surround it ${ }^{(2)}$.

The INV collapse is a common finding in patients who present with nasal airway obstruction. Although this can be a congenital condition, weakness of the internal nasal valve can also occur with nasal aging, following traumatic injury, and even more commonly because of prior nasal surgery secondary to rhinologic surgery ${ }^{(3)}$.

Correction of internal valve collapse must be done during functional rhinoplasty, which may include inferior turbinectomy, septoplasty, and placement of spreader grafts which is one of the most effective means of correcting internal valve collapse $^{(4)}$.

Poetker et al. ${ }^{(8)}$, measured the nasal valve angles of 30 patient by CT scans in 2 different planes in each patient. Coronal re-formations were performed in a plane perpendicular to the hard palate at the most anterior aspect of the nasal bones at the nasal dorsum. Re-formations of the NBV were performed in a plane perpendicular to the anterior aspect of the estimated acoustic axis. They found that the NBV may provide a more accurate assessment of the nasal valve, as the measured angles of the nasal valve in this plane were found to be more consistent with classic descriptions of $10^{\circ}$ to $15^{\circ}$.

Veron et al. ${ }^{(9)}$, discussed the CT scan for evaluation of INV at which 25 patients underwent analysis of surface and angle of each nasal valve by CT scans and predict if there is correlation between CT scans with clinical symptoms by (NOSE) score and showed that the best CT plan for studying of the nasal valve is probably perpendicular to the axis of nasal airflow and demonstrated the CT Scan analysis of the nasal valve does not correlate with the intensity of clinical symptoms as shown by (NOSE) scale.
Bloom et al. ${ }^{(6)}$, showed that there is no correlation between the pre-operatives modified Cottle maneuver scores for the INV angle and cross-sectional valve area values in reformatted CT scan orientation and traditionally oriented CT scans. Twenty-four rhinoplasty patients underwent a retrospective review of the pre-operative acquired reformatted $\mathrm{CT}$ scans to obtain sections through the INV and compared to pre-operative modified Cottle examination findings.

Orhan et al. ${ }^{(10)}$, studied 27 patients and found that (NOSE) scale is a very efficient tool in evaluating the outcomes of septoplasty.

Yeung et al. ${ }^{(11)}$, showed that the (NOSE) scale is very important to detect the improvement after nasal valve reconstruction with spreader grafts and alar grafts in which 79 patients evaluated by (NOSE) score pre and post rhinoplasty at 3 month assessment and showed there was significant improvement in the (NOSE) score pre and post rhinoplasty.

In our study, we included 20 rhinoplasty patients complaining of nasal obstruction due to nasal valve collapse and/or deviated nasal septum and/or inferior turbinate hypertrophy. Functional rhinoplasty was done with or without septoplasty with spreader graft insertion in most of the patients. The INV angle and cross-sectional area was measured radiologically by reformatted CT scans pre and 3 months post-rhinoplasty surgery. The reformatted CT scans done in a plane perpendicular to the anterior aspect of the acoustic axis which was based on the results of Poetker et al. ${ }^{(8)}$. As well, symptomatic evaluation was measured by (NOSE) scale pre and 3 months post-operative. In our study, we found that there was no statistically significant difference between pre and postoperative INV cross-sectional area, either on left side, right side or the average of two sides. As regard pre and post-operative INV angle, there is no statistically significant difference either on left side, right side or the average of two sides.

On the other hand, after evaluation of symptomatic improvement according to (NOSE) scale, there was a highly statistically significant difference between pre and post-operative nose score, with significant improvement in the scores after surgery which agrees with Orhan et al. ${ }^{(10)}$.

As well, there was non-significant correlation between CT scans post-operative INV angles and 
areas with post-operative (NOSE) score. So, there was no correlation between CT scans analysis of the INV and the (NOSE) scale which agrees with ${ }^{(9)}$.

\section{CONCLUSION}

There is high significant correlation between NOSE score pre and post-rhinoplasty. Therefore, the cross-sectional area and valve angle of the INV which was measured by reformatted CT scans is not of value in evaluation of the INV pre and post-rhinoplasty. However, NOSE score is of value to determine the degree of symptomatic improvement after rhinoplasty by comparing the score pre and post-rhinoplasty.

\section{REFERENCES}

1. Bailey B (1998): Nasal function and evaluation, nasal obstruction. Head and Neck Surgery: Otolaryngology. 2nd ed. New York, NY: Lippincott-Raven

2. Nigro CE, Nigo JF, Mino $\mathbf{O}$ and Mello JF (2009): Nasal valve: anatomy and physiology. Braz J Otorhinolaryngol., 75(2): 305-310.

3. Khosh M, Jen A, Honrado C and Pearlman $S$ (2004): Nasal valve reconstruction: experience in 53 consecutive patients. Archives of Facial Plastic Surgery, 6 (3): 167-71.

4. Kevin B (2011): Functional Rhinoplasty: The importance of the internal nasal valve. https://www.kevinbrennermd.com/blog/functio nal-rhinoplasty-the-importance-of-the-internalnasal-valve/424173/

5. Miman MC, Deliktas, H, Ozturan O, Toplu $Y$ and Akarc ay $M$ (2006): Internal nasal valve: re- visited with objective facts. Otolaryngol Head Neck Surg., 134(1): 41-47.

6. Bloom JD, Sridharan S, Hagiwara M, Babb JS, White WM and Constantinides $M$ (2012): Reformatted computed tomography to assess the internal nasal valve and association with physical examination. Arch Facial Plast Surg., 14(5): 331-5.

7. Çakmak Ö, Coşkun M, Çelik H, Büyüklü F, Özlüoğlu LN (2003): Value of acoustic rhinometry for measuring nasal valve area. The Laryngoscope, 113(2):295-302.

8. Poetker DM, Rhee JS, Mocan BO and Michel MA (2004): Computed tomography technique for evaluation of the nasal valve. Arch Facial Plast Surg., 6(4): 240-243.
9. Veron A, Bocquet J, Clareton V, Tourdias T, Molinier S, De Gabory $L$ and Dousset $V$ (2011): Bordeaux/FR. Value of CT scan measures of the nasal valve for predicting clinical nasal obstruction. European society of radiology. doi: 10.1594/ecr2011/C-1639.

10. Orhan KK, Miman MC, Yucel A, Yucedag F, Okur E, Altuntas A (2012): The efficiency of Nose Obstruction Symptom Evaluation (NOSE) scale on patients with nasal septal deviation. Auris Nasus Larynx, 39(3):275-9.

11. Yeung A, Hassouneh B, Kim DW (2016): Outcome of nasal valve obstruction after functional and aesthetic-functional rhinoplasty. JAMA facial plastic surgery, 18(2):128-34. 